\title{
ABSTRACT HYPERBOLIC CHAOS
}

\author{
AKHMET MARAT
}

\begin{abstract}
The abstract hyperbolic sets are introduced. Continuous and differentiable mappings as well as rate of convergence and transversal manifolds are not under discussion, and the symbolic dynamics paradigm is realized in a new way. Our suggestions are for more neat comprehension of chaos in the domain. The novelties can serve for revisited models as well as motivate new ones.
\end{abstract}

\section{INTRODUCTION}

Hyperbolic sets were introduced by S. Smale in famous papers [1,2]. They are fundamental for chaos theory, since describe profoundly domains, where the dynamics is happen. The genius of H. Poincaré, who explored the complexity in celestial mechanics [3], and scrupulous investigation of the Van der Pol oscillator by M. Cartright, J. Littlewood and H. Levinson [4, 5] caused the insights of S. Smail. Infinite number of hyperbolic points in a bounded area, symbolic dynamics, manifolds, Markov partitions and differentiable mappings make the background for the theory [6] - [15]

Recently, we have introduced a concept of domain structured chaos [16, 17, which may become a useful tool for analysis of complex dynamics [18]. This time, the new concept of abstract hyperbolic sets is considered to continue the line of the research. What is suggested, is not application of the symbolic dynamics through topological equivalence, which requests other advanced conditions for the relation establishment [9, 10, 12. Moreover, we do not utilize finite partitioning [7] and even countable [8] partitioning for involvement of the symbolic dynamics to explore the complexity. We perform the complete indexing of the domain. In other words, infinitesimal uncountable partitioning, and determine the map, which orbits are constructed through shifting in indexes such that the research can be focused on chaotic behavior. That is, a new realization of the Bernoulli map is present. By utilizing the separation and diameter conditions [17, 18, we explore the domain structured chaos in

2010 Mathematics Subject Classification. 65P20;34H10;60G05;60G10.

Key words and phrases. Abstract hyperbolic sets, Bi-infinite indexing, Chaotic stable set, Chaotic unstable set, Poincaré chaos, Li-Yorke chaos, Devaney chaos, Domain-structured chaos, Smale Horseshoe. 
the abstract hyperbolic sets. The discussion initiated in this paper can precise chaotic dynamics description as well as hyperbolic sets, explore relations, which either have not been seen in former studies or can provide more sense to them. For example, in the present paper, a new proof of the Smale horseshoe chaos is provided, which is free of the equivalence application. The results are complemented by Poincaré type of chaos and isolated dynamics on unstable sets.

\section{NONCHAOTIC DYNAMICS}

In this section we introduce a formal set and a map, which generalize what is is known for symbolic dynamics [14]. Another suggestion is about abstraction of the concept of hyperbolic sets [2,7], which is understood, in our research, as renouncing some of former properties and considering new ones, which are obvious, and useful.

Let the space $(\mathcal{F}, d)$ be given with metric $d$. The elements of the set are labeled such that

$$
\mathcal{F}=\left\{\mathcal{F}_{\ldots i_{-2} i_{-1} i_{0} . i_{1} i_{2} \ldots}: i_{k}=1,2, \ldots, m, k=\ldots-2,-1,0,1,2, \ldots\right\},
$$

where $m$ is a fixed natural number and the symbol . is used to determine the dynamics of the present research.

The set $\mathcal{F}$ is called an abstract hyperbolic set, and its elements are called hyperbolic points. The set of points,

$$
\mathcal{F}_{i_{1} i_{2} \ldots}=\left\{\mathcal{F}_{\ldots j_{-2} j_{-1} j_{0} . i_{1} i_{2} \ldots i_{n} \ldots}: j_{k}=1,2, \ldots, m, k=\ldots-2,-1,0\right\}
$$

is the stable set for a fixed point $\mathcal{F}_{\ldots i_{-k} \ldots i_{-2} i_{-1} i_{0} \cdot i_{1} i_{2} \ldots i_{n} \ldots}$, and the collection

$$
\mathcal{F}_{\ldots i_{-k} \ldots i_{-2} i_{-1} i_{0}}=\left\{\mathcal{F}_{\ldots i_{-2} i_{-1} i_{0} \cdot j_{1} j_{2} \ldots}: j_{k}=1,2, \ldots, m, k=1,2, \ldots\right\}
$$

is said to be the unstable set of the point.

The following unions are needed, in what follows,

$$
\mathcal{F}_{i_{-k} i_{-k+1} \ldots i_{0} . i_{1} i_{2} \ldots i_{n}}=\bigcup_{j_{k}=1,2, \ldots, m} \mathcal{F}_{\ldots i_{-k-1} i_{-k} i_{-k+1} \ldots i_{0} . i_{1} i_{2} \ldots i_{n} i_{n+1} \ldots},
$$

where indices $i_{-k}, i_{-k+1}, \ldots, i_{0}, i_{1}, i_{2}, \ldots, i_{n}$, are fixed,

$$
\mathcal{F}_{i_{1} i_{2} \ldots i_{n}}=\bigcup_{j_{k}=1,2, \ldots, m} \mathcal{F}_{\ldots i_{-k-1} i_{-k} i_{-k+1} \ldots i_{0} . i_{1} i_{2} \ldots i_{n} i_{n+1} \ldots},
$$

where indices $i_{1}, i_{2}, \ldots, i_{n}$, are fixed, and

$$
\mathcal{F}_{i_{-k} i_{-k+1} \ldots i_{0}}=\bigcup_{j_{k}=1,2, \ldots, m} \mathcal{F}_{\ldots i_{-k-1} i_{-k} i_{-k+1} \ldots i_{0}, i_{1} i_{2} \ldots i_{n} i_{n+1} \ldots},
$$

where indices $i_{-k}, i_{-k+1}, \ldots, i_{0}$, are fixed. 
We will say that the diameter condition is valid for the sets if for every fixed sequence $\ldots i_{-k} i_{-k+1} \ldots i_{0} \cdot i_{1} i_{2} \ldots i_{n} \ldots$ it is true that

$$
\operatorname{diam}\left(\mathcal{F}_{i_{-k} i_{-k+1} \ldots i_{0} . i_{1} i_{2} \ldots i_{n}}\right) \rightarrow 0 \text { as } k, n \rightarrow \infty,
$$

where $\operatorname{diam}(A)=\sup \{d(\mathbf{x}, \mathbf{y}): \mathbf{x}, \mathbf{y} \in A\}$, for a subset $A$ of $\mathcal{F}$.

Determine the following one-to-one and onto map $\varphi: \mathcal{F} \rightarrow \mathcal{F}$, such that

$$
\varphi\left(\mathcal{F}_{\ldots i_{-k} \ldots i_{-2} i_{-1} i_{0} . i_{1} i_{2} \ldots i_{n} \ldots}\right)=\mathcal{F}_{\ldots i_{-k} \ldots i_{-2} i_{-1} i_{0} i_{1} . i_{2} \ldots i_{n} \ldots} .
$$

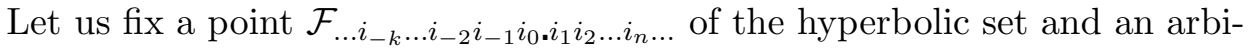
trary element, $\mathcal{F}_{\ldots j_{-2} j_{-1} j_{0} i_{1} i_{2} \ldots i_{n} \ldots}$, from the stable manifold of the point. The definition of the stable set and the diameter property it follows that

$$
d\left(\varphi^{n}\left(\mathcal{F}_{\ldots j_{-2} j_{-1} j_{0} . i_{1} i_{2} \ldots i_{n} \ldots}\right), \varphi^{n}\left(\mathcal{F}_{\ldots i_{-k} \ldots i_{-2} i_{-1} i_{0} . i_{1} i_{2} \ldots i_{n} \ldots}\right)\right) \rightarrow 0
$$

as $n \rightarrow \infty$.

Similarly, it is true that

$$
d\left(\varphi^{n}\left(\mathcal{F}_{\ldots i_{-2} i_{-1} i_{0} \cdot j_{1} j_{2} \ldots j_{n} \ldots}\right), \varphi^{n}\left(\mathcal{F}_{\ldots i_{-k} \ldots i_{-2} i_{-1} i_{0} . i_{1} i_{2} \ldots i_{n} \ldots}\right)\right) \rightarrow 0
$$

as $n \rightarrow-\infty$, for points of the unstable manifold.

It is clear that

$$
\mathcal{F} \supseteq \mathcal{F}_{i_{1}} \supseteq \mathcal{F}_{i_{1} i_{2}} \supseteq \ldots \supseteq \mathcal{F}_{i_{1} i_{2} \ldots i_{n}} \supseteq \mathcal{F}_{i_{1} i_{2} \ldots i_{n} i_{n+1}} \ldots
$$

and

$$
\mathcal{F} \supseteq \mathcal{F}_{i_{0}} \supseteq \mathcal{F}_{i_{-1} i_{0}} \supseteq \ldots \supseteq \mathcal{F}_{i_{-n} i_{-n+1} \ldots i_{0}} \supseteq \mathcal{F}_{i_{-n-1} i_{-n} \ldots i_{0}} \ldots
$$

Considering iterations of the map, one can verify that

$$
\varphi^{n}\left(\mathcal{F}_{i_{1} i_{2} \ldots i_{n}}\right)=\mathcal{F}
$$

and

$$
\varphi^{-n}\left(\mathcal{F}_{i_{-n} i_{-n+1} \ldots i_{0}}\right)=\mathcal{F},
$$

for arbitrary natural number $n$. The relations (2.8) and (2.9) give us a reason to call $\varphi$ a similarity map and the number $n$ the order of similarity. We called it abstract similarity map in [18].

\section{Chaos}

Denote by $d(A, B)=\inf \{d(\mathbf{x}, \mathbf{y}): \mathbf{x} \in A, \mathbf{y} \in B\}$ the function of two bounded subsets $A$ and $B$ of $\mathcal{F}$. The space $(\mathcal{F}, d)$ satisfies the separation condition of degree $n$ if there exist a positive number $\varepsilon_{0}$ and a natural number $n$ such that for arbitrary indices $i_{1} i_{2} \ldots i_{n}$ one can find indices $j_{1} j_{2} \ldots j_{n}$ such that

$$
d\left(\mathcal{F}_{i_{1} i_{2} \ldots i_{n}}, \mathcal{F}_{j_{1} j_{2} \ldots j_{n}}\right) \geq \varepsilon_{0} .
$$

A point $\mathcal{F}_{\ldots i_{-k} \ldots i_{-2} i_{-1} i_{0} . i_{1} i_{2} \ldots i_{n} \ldots}$ from $\mathcal{F}$ is periodic with period $n$ if its index consists of endless repetitions of a block of $n$ terms. 
Theorem 1. If the diameter and separation conditions are valid, then the similarity map $\varphi$ is chaotic on each unstable set of the dynamics in the sense of Devaney .

Proof. Utilizing the diameter condition, the transitivity will be proved if there exists an element $\mathcal{F}_{\ldots i_{-k} \ldots i_{-2} i_{-1} i_{0} i_{1} i_{2} \ldots i_{n} \ldots}$ of the unstable set such that for any finite sequence $j_{-k} \ldots j_{-2} j_{-1} j_{0} j_{1} j_{2} \ldots j_{n}$ one can find that for a sufficiently large $p$, the point $\varphi^{p}\left(\mathcal{F}_{\ldots i_{-k} \ldots i_{-2} i_{-1} i_{0} . i_{1} i_{2} \ldots i_{n} \ldots}\right)$ belongs to $\mathcal{F}_{j_{-k} \ldots j_{-2} j_{-1} j_{0} \cdot j_{1} j_{2} \ldots j_{n}}$. This is true, since one can construct a sequence $\ldots i_{1} i_{2} \ldots i_{n} \ldots$, which contains all the sequences of the type $j_{-k} \ldots j_{-2} j_{-1} j_{0} j_{1} j_{2} \ldots j_{n}$ as blocks.

Fix a member $\mathcal{F}_{\ldots i_{-k} \ldots i_{-2} i_{-1} i_{0} i_{1} i_{2} \ldots i_{n} \ldots}$ of the unstable set and a positive number $\delta$. Find a sufficiently large number $k \operatorname{such}$ that $\operatorname{diam}\left(\mathcal{F}_{i_{1} i_{2} \ldots i_{k}, i_{1} i_{2} \ldots i_{k}}\right)<$ $\delta$ and choose a $k$-periodic element $\mathcal{F}_{\ldots i_{1} i_{2} \ldots i_{k}, i_{1} i_{2} \ldots i_{k} \ldots}$ of $\mathcal{F}_{i_{1} i_{2} \ldots i_{k}}$. It is clear that the periodic point is an $\delta$-approximation for the considered member. Thus, the density of periodic points is proved.

For sensitivity, fix a point $\mathcal{F}_{\ldots i_{-k} \ldots i_{-2} i_{-1} i_{0}, i_{1} i_{2} \ldots i_{n} \ldots}$ of the unstable set and an arbitrary positive number $\varepsilon$. Due to the diameter condition there exist an integer $k$ and point $\mathcal{F}_{\ldots i_{-k} \ldots i_{-2} i_{-1} i_{0} . i_{1} i_{2} \ldots i_{k} j_{k+1} j_{k+2} \ldots} \neq \mathcal{F}_{\ldots i_{-k} \ldots i_{-2} i_{-1} i_{0}, i_{1} i_{2} \ldots i_{n} \ldots}$ such

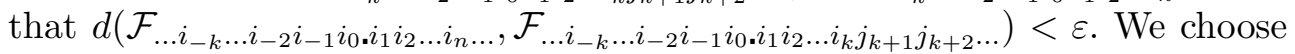
$j_{k+1}, j_{k+2}, \ldots$ such that $d\left(\mathcal{F}_{i_{k+1} i_{k+2} \ldots i_{k+n}}, \mathcal{F}_{j_{k+1} j_{k+2} \ldots j_{k+n}}\right)>\varepsilon_{0}$, by the separation condition. This proves the sensitivity.

In [19, Poisson stable motion is utilized to distinguish chaotic behavior from periodic motions in Devaney and Li-Yorke types. The dynamics is given the named Poincaré chaos. The next theorem shows that the Poincaré chaos is valid for the similarity dynamics.

Theorem 2. The abstract similarity map $\varphi$ is chaotic in Poincaré sense on each unstable set of the dynamics, if the diameter and separation, properties are valid.

The proof of the last theorem is based on the verification of Lemma 3.1 in [19], applied to the similarity map.

In addition to the Devaney and Poincaré chaos, it can be shown that the Li-Yorke chaos exists fo map $\varphi$ on every unstable set of the dynamics. The proof is similar to that of Theorem 6.35 in [20]. Thus, we have obtained that there are Poncaré, Li-Yorke and Devaney chaos on each unstable set of the similarity map. Consequently, the hyperbolic set $\mathcal{F}$ admits the domain structured chaos. It is important to say that the dynamics is the union of chaotic unstable sets. Moreover, the chaos can be approved by considering bi-infinite symbolic dynamics, but we decide to utilize the above set-by-set way, since it provides more information on the structure of the chaos. It is clear, also, that accepting the separation property for decreasing time, one can approve domain structured chaos for stable sets. 


\section{Smale horseshoes}

As an example, consider the Smale horseshoe map [1. We will discuss the dynamics omitting many details, which can be found in [9]. The Smale horseshoe map is the set of basic topological operations of stretching and folding. The result of the iterations are invariant sets

$$
\Lambda_{+}=\left\{q \in S: F^{k}(q) \in S, k=1,2, \ldots\right\}
$$

and

$$
\Lambda_{-}=\left\{q \in S: F^{-k}(q) \in S, k=1,2, \ldots\right\},
$$

where $S$ is the unit square and $F$ is the Smale map. Sets $\Lambda_{+}$and $\Lambda_{-}$are products of Cantor sets with a vertical and a horizontal lines respectively. The domain under discussion is $\Lambda=\Lambda_{+} \cap \Lambda_{-}$. In literature, the dynamics $(\Lambda, F)$ is proved to be topologically equivalent to the dynamics of $\left(\Sigma_{2}, \sigma\right)$, where $\Sigma_{2}$ is the set of two-sided sequences of $0^{\prime} \mathrm{s}$ and $1^{\prime}$ s and $\sigma$ is the Bernoulli shift. Considering the equivalence, chaos was proved for the map $F$. In our case, to accomplish the analysis we utilize the experience just for the indexing points of the set $\Lambda$, and constructing the abstract hyperbolic set $\mathcal{F}$ with the Euclidean planar distance. The construction in [9] demonstrates that the diameter and separation conditions are valid for the abstract hyperbolic set. Consequently, the domain structured chaos of the similarity map is proper for the set $\Lambda$. Apparently, the way of chaos generation can be applied to other sets with hyperbolic structure [6]- [15].

\section{Conclusion}

We suggest an abstraction for two-sided dynamical processes with discrete time. A method of chaos generation is provided as dynamics of the abstract similarity map. Thus, all the three types of Poincaré, Li-Yorke and Devaney chaos are approved in the domain. The approach is also of strong practical sense. For instance, it has been realized in our papers [17, 18] for fractals and neural networks. This time, it is proven that the hyperbolic set is a union of chaotic unstable sets. Thus, comprehension of the dynamical has been deepen. It is important that the chaotic behavior can be extend for the decreasing time, if one assume the similar separation condition. Another benefit is that multidimensional generalizations are possible on the basis of proposals. The processes in the focus are deterministic. Not only the deep past, but the very far future has to be determined for the abstract similarity map application. That is, the hyperbolic dynamics considers processes with infinite chaotic history. Nevertheless, the approach does not exclude possibility of random dynamics description [16]. 


\section{REFERENCES}

[1] Smale, S., Diffeomorphisms with many periodic points, In Differential and Combinatorial Topology: A Symposium in Honor of Marston Morse, Princeton University Press, Princeton, 63-70 (1966).

[2] Smale, S., Differentiable dynamical systems, Bull. Am. Math. Soc., 73, 747-817 (1967).

[3] Poincaré, H., New Methods of Celestial Mechanics Volume I-III, Dover Publications, New York, NY (1957).

[4] Cartwright, M., Littlewood, J., On nonlinear differential equations of the second order I: the equation $y^{\prime \prime}-k\left(1-y^{2}\right) y^{\prime}+y=b k \cos (\lambda t+a), k$ large, J. Lond. Math. Soc., 20, 180-189 (1945).

[5] Levinson, N., A second order differential equation with singular solutions, Ann. Math., 50, 127-153 (1949).

[6] Adler, R., Konheim, A. and McAndrew, M., Topological entropy.Trans. Amer. Math. Soc.,114 309-319 (1965).

[7] Bowen, R., Markov partitions for axiom A diffeomorphisms, Am. J. Math., 92, 725747 (1970)

[8] Bunimovich, L., Sinai, Ya., Markov Partitions for Dispersed Billiards, Commun. Math. Phys., 78 (2), 247280 (1980).

[9] Devaney, R. L., An Introduction to Chaotic Dynamical Systems. Addison-Wesley, Menlo Park, (1987).

[10] Guckenheimer, J., Holmes, P.J., Nonlinear oscillations, Dyanamical Systems and Bifurcations of Vector Fields, Springer, New York (1997).

[11] Eckmann J.P. and D. Ruelle, D., Ergodic theory of chaos and strange attractors, Rev. Mod. Phys., 57, 617656 (1985).

[12] Newhouse S., Lectures on Dynamical Systems, Progress in Mathematics, Birkhauser, New York (1980).

[13] Ruelle, D., An inequality of the entropy of differentiable maps, Bol. Soc. Bras. Mat., 9, 8387 (1978).

[14] Wiggins, S., Global Bifurcation and Chaos: Analytical Methods, Springer-Verlag, New York, Berlin (1988).

[15] Williams, S. (editor), Symbolic Dynamics and its Applications. Proc. Symp. Appl. Math. 60 (2004).

[16] M. Akhmet, 2019, Domain-structured chaos in discrete random processes, Arxiv eprints, arXiv:1912.10478 (submitted).

[17] M. Akhmet, E. M. Alejaily, 2019, Domain-Structured Chaos in a Hopfield Neural Network, Int. J. Bifurc. Chaos, 29 (14), 1950205.

[18] M. Akhmet, E. M. Alejaily, 2020, Abstract similarity, chaos and fractals, Discrete and Continuous Dynamical Systems, Ser. B. ( in press) doi:10.3934/dcdsb.2020191

[19] Akhmet, M. and Fen, M. O. 2016, Poincar chaos and unpredictable functions. Commun. Nonlinear Sci. Numer. Simulat. 48 85-94.

[20] G. Chen, Y. Huang, 2011, Chaotic Maps: Dynamics, Fractals and Rapid Fluctuations, Synthesis Lectures on Mathematics and Statistics, Morgan and Claypool Publishers, Texas.

Middle East Technical University, Department of Mathematics, Ankara, Turkey

E-mail address: marat@metu.edu.tr 